\title{
„Wie auf einem schwankenden Schiff“ - ein Fallbeispiel
}

Frau Meier ${ }^{1}$ stürzt vom Rad. Zuerst scheint außer einer Fingerfraktur alles in Ordnung zu sein. Doch nach zwei Wochen treten mehrmals täglich Schwindelattacken auf, die Frau Meier sehr im Alltag einschränken. Eine multimodale physiotherapeutische Therapie, die auf klaren, einem Clinical Reasoning Prozess folgenden Hypothesen aufbaut, verringert den Schwindel zu 100 Prozent.

\section{Vorgeschichte}

Frau Meier, 59 Jahre alt, stellt sich auf Empfehlung ihrer Tochter zur Physiotherapie vor. Sie war vor einem Jahr mit dem Fahrrad gestürzt und hatte sich dabei eine offene Finger-Luxationsfraktur und ein Hämatom über dem linken Auge zugezogen. Nachdem der Finger sofort nach dem Unfall erfolgreich operiert worden war, trat zwei Wochen posttraumatisch plötzlich ein anfallsartiger Schwankschwindel mit einer Dauer von je drei bis fünf Sekunden auf. Da der Schwindel sich nicht verbesserte, suchte Frau Meier nach einem halben Jahr ihren Hausarzt auf. Dieser überwies sie nach Abklärung möglicher medizinischer Ursachen an den HalsNasen-Ohren Arzt, der durch Tests einen Lagerungsschwindel ausschloss und Medikamente verordnete, welche die Durchblutung des Innenohrs verbessern und so den Schwindel unspezifisch beeinflussen sollten. Der Schwindel reduzierte sich durch diese medikamentöse Behandlung jedoch nicht. Frau Meier nimmt diese Medikamente trotzdem weiterhin und hat inzwischen Angst, dass der Schwindel andere, gefährliche Ursachen haben könnte. Der Schwindel hat sich in letzter Zeit nicht verändert und tritt nun täglich mindestens achtmal auf. Frau Meier kommt mit dem Anliegen in die Physiotherapie, abzuklären, ob der Schwindel eventuell durch eine Therapie des Nackens beeinflusst werden kann.

\section{Subjektiver Befund \\ Hauptproblem}

Frau Meier gibt den anfallsartigen Schwindel bei verschiedenen Kopfbewegungen und Lageveränderungen als ihr Hauptproblem an. Ihr Schwindel fühlt sich so an, als ob sie auf einem schwankenden Schiff sei.

\section{Symptomauslösung/24-Stunden-Verhalten}

- Schnelles Hinlegen und Aufsitzen aus der Rücken- und Seitlage sowie Drehen im Bett lösen sofort den Schwindel für eine Dauer von drei bis fünf Sekunden aus ( $\bullet$ Abb. 1 (1)).

- Nach unten und nach oben Schauen lösen manchmal den Schwindel aus ( Abb. 1 (1)).

- Im Stand das Fahrradschloss Öffnen (Kopf nach unten und gedreht) löst sofort den Schwindel für drei bis fünf Sekunden aus ( Abb. 1 (1)).

1 Name von der Redaktion geändert

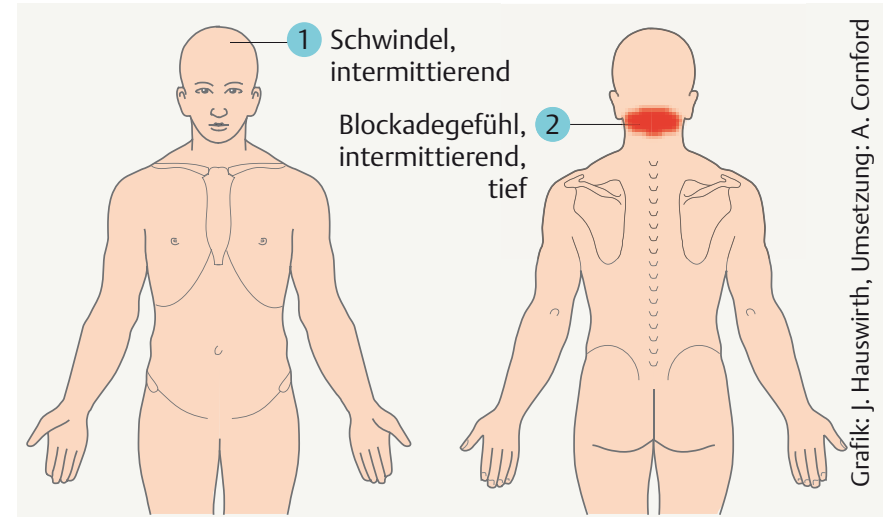

Abb. 1 Körpertabelle.

- Der Schulterblick nach rechts im Auto löst sofort den Schwindel für drei bis fünf Sekunden aus ( Abb. 1 (1).

- Der Schwindel wird bei körperlich anspruchsvollen Tätigkeiten wie Bergauffahren mit dem Fahrrad oder Hausarbeiten wie Staubsaugen verstärkt ( Abb. 1 (1)).

- Frau Meier vermeidet das Wandern in den Bergen sowie steile Treppen, da sie Angst hat, aufgrund des Schwindels zu stürzen. Ausserdem fühlt sie sich in ihrer beruflichen Tätigkeit in der Erwachsenenbildung beim schnellen Aufstehen vom Sitz beeinträchtigt, da dies auch den Schwindel auslösen kann.

\section{Zusätzliche Symptome}

- Gefühl von Steifigkeit bei unterschiedlichen Bewegungen des Nackens seit dem Unfall ( $\bullet$ Abb. 1 (2))

- unspezifische Nackenschmerzen dreimal pro Monat für je zwei Tage, die schon vor dem Fahrradunfall auftraten $(\bullet$ Abb. 1 (2)

- migräneartige Kopfschmerzen ein- bis zweimal pro Monat, die schon vor dem Fahrradunfall auftraten

\section{Spezielle Fragen}

- keine Übelkeit, Seh-, Schluck- oder Sprachprobleme, kein plötzliches Einsinken der Beine

- keine Taubheit oder Kribbeln im Gesichtsbereich und keine subjektive Muskelschwäche

- kein Übergewicht; stabile Gewichtssituation 
- Blutdruck normal

- Nichtraucherin

- Medikamente: nur durchblutungssteigerndes Medikament für Innenohr vom HNO-Arzt

- keine weiteren Erkrankungen

- keine frühere Tumorerkrankung

- keine Osteoporose

- Röntgen nach dem Unfall zeigte keine Fraktur der HWS oder des Schädels.

\section{Erste Hypothesenbildung}

Nach dem subjektiven Befund versuche ich, zwischen drei möglichen Hypothesen zu unterscheiden $(\mathbf{A b b} . \mathbf{2}$ und $\bullet$ Tab. 1). Zudem kläre ich in der anschließenden Funktionsuntersuchung mögliche Kontraindikationen wie eine hochzervikale Instabilität (Integrität der Ligg. alaria und des Lig. transversum atlantis) oder eine VBI/CAD (Vertebrobasiläre Insuffizienz/Cerviko-Arterielle Dysfunktion) [9] ab. Anhand der funktionellen Demonstration, der Analyse der auffälligen Zeichen und Symptome sowie der Durchführung des Dix Hallpike Manövers versuche ich, zwischen zervikogener und vestibulärer Schwindelursache zu differenzieren.

\section{Funktionsuntersuchung: Inspektion}

- Kopf steht in einer leichten ventralen Translation. Korrektur der Position reproduziert keine Symptome.

- prominenter C7 mit vermehrter Kyphose BWS

- sichtbare Faltenbildung auf Höhe C5

\section{Funktionelle Demonstration}

Der Transfer aus der Rückenlage in den Sitz löst den Schwindel bei Frau Meier sofort für fünf Sekunden mit einer Intensität von 5/10 VAS aus $[5,16]$. Während des Transfers tritt weder ein Nystagmus auf noch sind sonstige zusätzliche Symptome zu erkennen. Eine zusätzliche manuelle Stabilisation der HWS verbessert den Schwindel auf 2/10 VAS ( Abb. 3 ).

\section{Hochzervikale Instabilitätstests}

Nach einem Sturz vom Fahrrad mit anschließendem Schwindel ist es sinnvoll, zuerst die obere HWS bezüglich einer ligamentären Instabilität zu untersuchen.

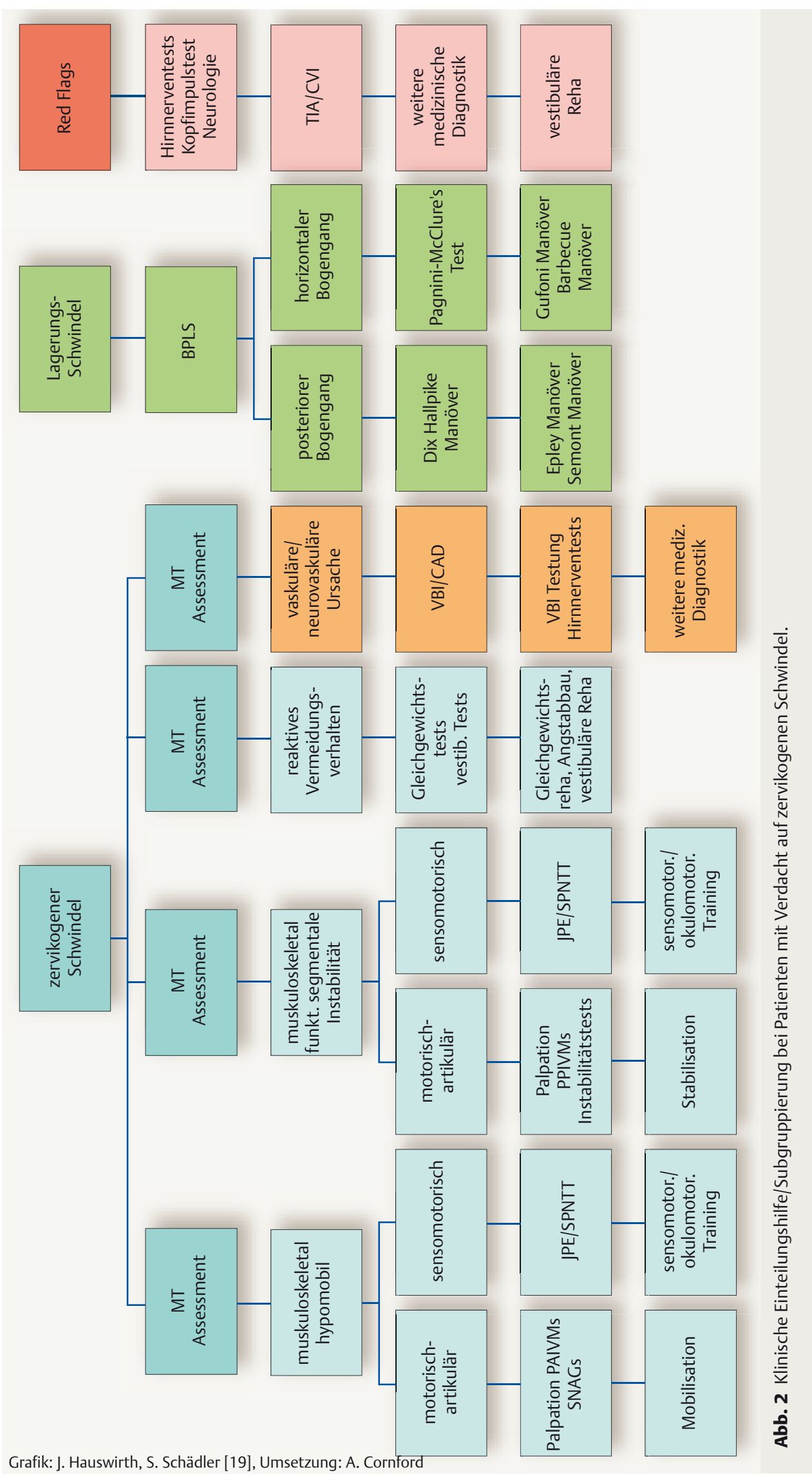


Tab. 1 Differentialdiagnostik.

\begin{tabular}{|c|c|c|}
\hline & Pro & Kontra \\
\hline $\begin{array}{l}\text { Lagerungsschwindel } \\
\text { BPLS (Benigner Paroxys- } \\
\text { maler Lagerungsschwin- } \\
\text { del)/vestibulär }\end{array}$ & $\begin{array}{l}\text { - lagewechselabhängig } \\
\text { - Ermüdbarkeit des Schwindels }\end{array}$ & $\begin{array}{l}\text { - } \text { sofort bei Bewegung } \\
\text { - } \text { kein Drehschwindel } \\
\text { - } \text { auftretend ohne kurze Verzögerung } \\
\text { - HNO Abklärung }\end{array}$ \\
\hline Zervikogener Schwindel & $\begin{array}{l}\text { - Schwankschwindel } \\
\text { - bewegungsabhängig } \\
\text { - Beginn nach Trauma } \\
\text { - Nackenschmerzen in Anamnese }\end{array}$ & - kurze Attacken von Schwindel \\
\hline $\begin{array}{l}\text { VBI (Vertebrobasiläre } \\
\text { Insuffizienz) als Red Flag }\end{array}$ & - Zunahme bei körperlicher Aktivität & $\begin{array}{l}\text { - } \text { keine Blutdruckprobleme } \\
\text { - } \text { keine zusätzlichen Probleme wie Übelkeit, Visus- oder Schluckproble- } \\
\text { me, Taubheit usw. } \\
\text { - } \text { keine Veränderung über ein Jahr: z. B. keine neurologischen Zeichen } \\
\text { - Schwindel verstärkt sich nicht, wenn Position gehalten wird } \\
\text { - } \text { kein Hinweis auf eine vorliegende bekannte Gefässpathologie }\end{array}$ \\
\hline
\end{tabular}

3
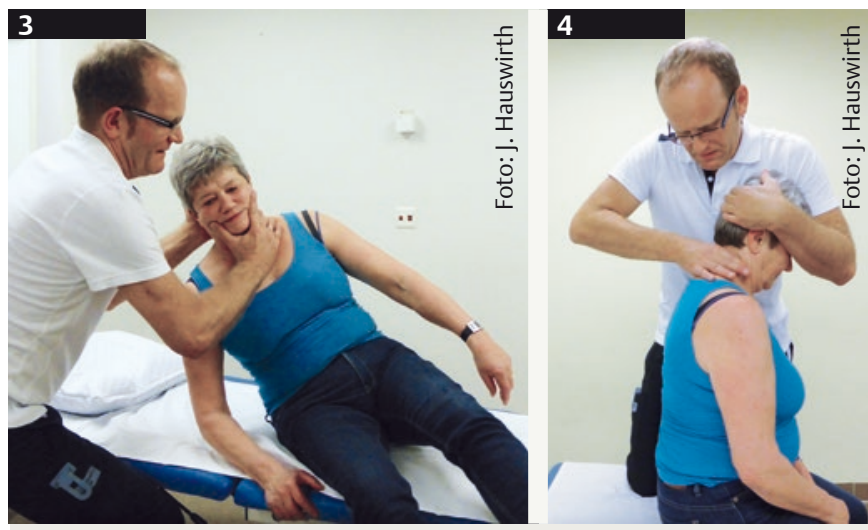

Abb. 3 Transfer aus Rückenlage in den Sitz mit manueller Stabilisation der HWS.

Abb. 4 Sharp-Purser Test in Flexion im Sitz.

Der Sharp-Purser Test [25] untersucht die Integrität des Lig. transversum. Die Flexion der Halswirbelsäule löst bei Frau Meier den typischen Schwindel aus. Ich fixiere in der Flexionsposition der HWS den Dornfortsatz des Axis und schiebe das Occiput nach dorsal. Den Test führe ich nochmals in Rückenlage aus. Frau Meier spürt keine Veränderung des Schwindels - weder im Sitz noch in Rückenlage. Das Endgefühl ist hart. Diese Resultate sprechen gegen eine Insuffizienz des Lig. transversum ( $\bullet$ Abb. 4 ).

Die Integrität der Ligg. alaria untersuche ich, indem ich den Dornfortsatz des Axis fixiere und gleichzeitig die Halswirbelsäule rotiere und lateral flektiere [25]. Die Lateralflexion läuft sofort auf den Dornfortsatz weiter, was für eine gute Integrität der Ligg. alaria spricht. Somit besteht kein Verdacht auf eine hochzervikale ligamentäre Instabilität. Eine Untersuchung der oberen Halswirbelsäule mit PPIVMs in Lateralflexion bestätigt diese Hypothese zusätzlich.

\section{Differenzierung zervikale und vestibuläre Schwindelursache}

Das Dix Hallpike Manöver verursacht bei Frau Meier einen Schwindel, jedoch ohne Übelkeit und Nystagmus. Bei guter manueller Stabilisation des Nackens verbessert sich der Schwindel deutlich $(\downarrow$ Abb. 5). Mit Wiederholung des Tests verbessert sich der Schwindel

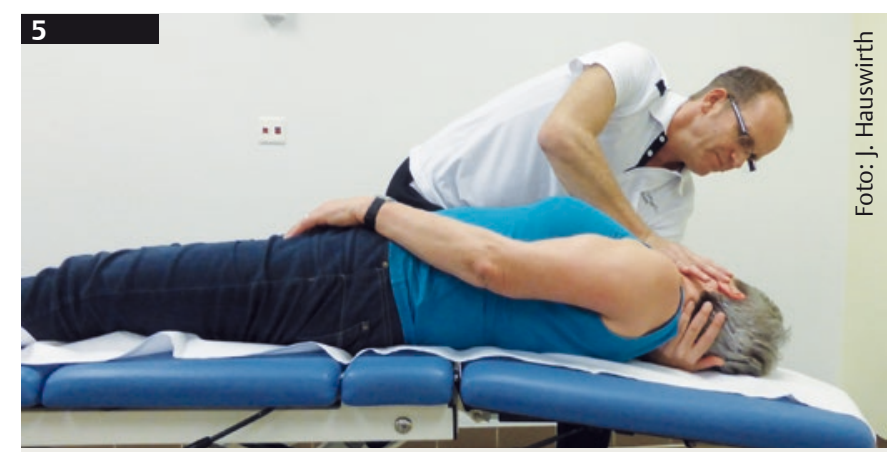

Abb. 5 Dix Hallpike Manöver mit zusätzlicher Stabilisation der HWS.

Tab. 2 Aktive Bewegungen HWS.

\begin{tabular}{|l|l|}
\hline Bewegungsrichtung & Range of Motion, Symptome \\
\hline Flexion & $40^{\circ},(1)+$ \\
\hline Extension & $20^{\circ}$, (1), Knick in der mittleren HWS \\
\hline Rotation links & $70^{\circ}, \square$ \\
\hline Rotation rechts & $50^{\circ},(1)$ \\
\hline Lateralflexion links & $10^{\circ}$, (2) + Diff.: obere Cx(2) + \\
\hline Lateralflexion rechts & $30^{\circ}$, , \\
\hline
\end{tabular}

ebenfalls. Die vestibuläre Hypothese ist dadurch noch nicht ganz ausgeschlossen, da eine Verbesserung des Schwindels bei Wiederholung des Tests bei einer vestibulären Ursache möglich ist. Die Verbesserung durch manuelle Stabilisation und das Fehlen eines Nystagmus deuten aber eher auf ein zervikales Instabilitätsproblem.

\section{Differenzierung obere/mittlere/untere HWS}

Nach den aktiven HWS Bewegungen besteht die Hypothese, dass eher die obere HWS betroffen ist, da Frau Meier ein gegensinnig gekoppeltes Bewegungsmuster zeigt, das für die obere HWS als Ursache spricht. Zusätzlich wird bei der Differenzierung in Lateralflexion die obere HWS als symptomverstärkend angezeigt ( $\bullet$ Tab. 2). 

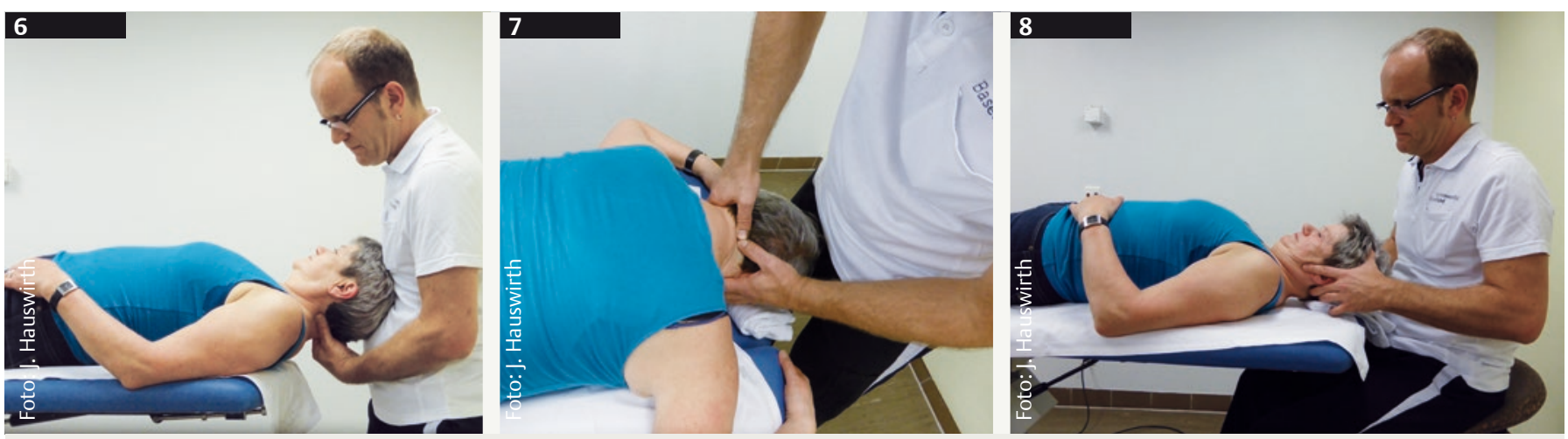

Abb. 6 Passive Physiological Intervertebral Movements (PPIVMs nach Maitland et al. 2001 [12]) für Rotation zwischen Okziput und C2.

Abb. 7 Posterior-anteriore Bewegung unilateral C1 rechts.

Abb. 8 Kraniozervikaler Flexionstest (CCFT).

Tab. 3 Ursachen-Hypothesenbildung vor der weiteren Behandlung

\begin{tabular}{|l|l|l|}
\hline & Pro & Kontra \\
\hline $\begin{array}{l}\text { Zervikogener Schwindel } \\
\begin{array}{l}\text { muskuloskeletal } \\
\text { hypomobile Ursache }\end{array}\end{array}$ & $\begin{array}{l}\text { - Beweglichkeitsdefizit der oberen HWS } \\
\text { - Beeinflussung des Schwindels durch Mobilisation C1-C3 }\end{array}$ & $\begin{array}{l}\text { Schwindel konnte durch eine Veränderung der } \\
\text { Beweglichkeit nicht ganz beseitigt werden }\end{array}$ \\
\hline $\begin{array}{l}\text { Zervikogener Schwindel } \\
\text { muskuloskeletal } \\
\text { hypermobil/funktionelle } \\
\text { segmentale Instabilität }\end{array}$ & $\begin{array}{l}\text { - Verbesserung der Schwindelproblematik in der funktionel- } \\
\text { len Demonstration und beim Dix Hallpike Manöver durch } \\
\text { manuelle Stabilisation der HWS durch den Therapeuten }\end{array}$ & $\begin{array}{l}\text { bisher in der Funktionsuntersuchung noch } \\
\text { keine Hypermobilität aufgefunden }\end{array}$ \\
\hline Knick bei C5 in der Inspektion und bei HWS Extension
\end{tabular}

Folgende Tests unterstützen diese Hypothese zusätzlich:

- Passive Rotation C0/C1 bei manuell fixiertem Axis: PROM (Passive Range of Motion) nach rechts nur fünf Grad, nach links 25 Grad ( Abb. 6). Dies bestätigt die Hypothese, dass ein grosser Anteil des Rotationsdefizits durch die obere HWS verursacht wird [25].

- Flexionsrotationstest: In Flexion rotiert die HWS nach links um 20 Grad und nach rechts um fünf Grad [2].

\section{Erste Behandlung}

In der ersten Behandlung überprüfe ich die obere HWS mittels Palpation und passiver Untersuchung weiter auf Symptome und Zeichen und verfolge das Ziel, mit einer Probebehandlung die vorläufige Hypothese zu bestätigen oder zu verwerfen.

\section{Passive Untersuchung der oberen HWS}

Palpation in Bauchlage inklusive PAIVMs (Passive Accessory Intervertebral Movements nach Maitland et al. 2001 [12]):

- Haut-Abhebbarkeit im Bereich von C1 verringert

- subokzipitale Muskulatur rechts sehr fest

- C1 posterior-anteriore Gleitbewegung unilateral rechts mit sehr viel Widerstand im Seitenvergleich und schmerzhaft:(2) auslösbar $($ Abb. 1)

- C2 posterior-anteriore Gleitbewegung unilateral rechts sehr steif ohne Symptome

- C3 symptomfrei und deutlich bessere Beweglichkeit

\section{Probebehandlung}

- C1-C2 rechts unilaterale posterior-anteriore Gleitbewegung mit Grad III nach Maitland [12] in den Widerstand und mit leichter Schmerzauslösung ( $\bullet$ Abb. 1 (2)) für 3 Minuten ( $\bullet$ Abb. 7)
Der Wiederbefund der Beweglichkeit zeigt eine verbesserte HWS Beweglichkeit in Flexion und in Rotation nach rechts um $20 \mathrm{Grad}$ und einen verringerten Schwindel auf 3/10.

Hypothese nach der Probebehandlung. Durch die erste Probebehandlung der oberen HWS unilateral rechts kann das einseitig vorhandene Beweglichkeitsdefizit der HWS verbessert werden. Gleichzeitig verändert sich das Hauptsymptom der Patientin, der Schwindel ( Abb. 1 (1)), und das Blockadegefühl subokzipital ( Abb. 1 (2)). Somit bestätigt diese Probebehandlung, dass der Schwindel zum Teil zervikogen verursacht wird.

Nach der Probebehandlung füllt Frau Meier den Schwindelfragebogen Dizziness Handicap Inventory-German Version (DHI-G) aus [18]. Die Auswertung dieses Fragebogens, der die Auswirkung des Schwindels auf funktionelle, emotionale und physische Aspekte des Alltags erfasst [11], ergibt bei Frau Meier eine Alltagseinschränkung von 32/100 Punkten.

\section{Therapieplanung und -verlauf}

1. Weiterbehandlung des Bewegungsdefizits hochzervikal

2. Ursachen-Hypothesenbildung vor der weiteren Behandlung $(\triangleright$ Tab. 3)

3. Untersuchung der mittleren/unteren HWS bezüglich funktioneller Instabilität

\section{Zweite und dritte Behandlung}

Weiterhin mobilisiere ich manuell C1-C2 mit steigender Intensität und mittels Vorpositionierung des Kopfes in Rotation nach rechts. Zudem untersuche ich die segmentale Beweglichkeit der mittleren/unteren HWS (C3-C7) mittels Passive Physiological Intervertebral Movements (PPIVMs) und überprüfe so eine denkbare Hy- 


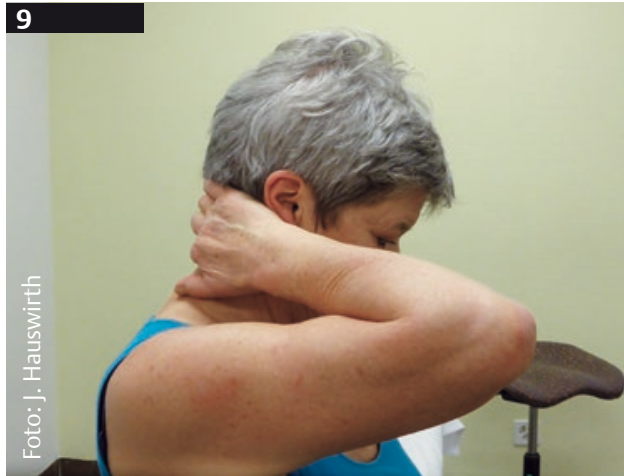

permobilität oder Instabilität. Die Untersuchung ergibt ein hypermobiles Segment in Flexion auf Höhe C5/6. Zudem setzt Frau Meier beim kraniozervikalen Flexionstest (CCFT) [8] anstatt der tiefen Nackenflexoren die oberflächliche Muskulatur, insbesondere den M. sternocleidomastoideus, exzessiv ein ( $\mathbf{A b b} \mathbf{b}$ ).

Hypothesenbildung nach drei Behandlungen. Durch die hochzervikalen Behandlungstechniken ist die Rotations- und Lateralflexionsbewegung im Seitenvergleich nun ausgeglichen und die Schwindelhäufigkeit im Alltag um 40 Prozent reduziert. Auch die Intensität des Schwindels ist positiv verändert auf $2 / 10$. Um das nach der Behandlung verbesserte Bewegungsausmaß längerfristig zu erhalten, erhält die Patientin ein Heimprogramm mit gehaltenen natürlichen Gleitbewegungen auf Höhe $\mathrm{C} 2$ von posterior nach anterior (Mobilisation mit Bewegung: SNAGs [14]) ( Abb. 9). Die Patientin führt diese Eigenmobilisationen sechsmal nacheinander mindestens dreimal am Tag in Kombination mit zervikaler Flexion konsequent zu Hause aus und kann die Schwindelhäufigkeit dadurch nochmals um 20 Prozent reduzieren.

In der funktionellen Demonstration zu Beginn konnte der Schwindel beim Aufsitzen durch manuelle Stabilisation der HWS reduziert werden. Dieser Befund sowie die vergrößerte passive segmentale Beweglichkeit auf Höhe C5/6 und das motorische Kontrolldefizit beim CCFT verstärken die Hypothese einer verringerten Stabilisationsfähigkeit der HWS, die möglicherweise zum Schwindel beiträgt.

\section{Vierte bis sechste Behandlung}

Aufgrund der Hypothese der verringerten Stabilisationsfähigkeit der HWS trainiert Frau Meier in der vierten, fünften und sechsten Behandlung die tiefen Nackenflexoren in Rückenlage und beim Transfer vom Liegen ins Sitzen. Nach dieser Behandlungsserie sind nur noch 20 Prozent der Schwindelsymptomatik im Alltag vorhanden.

Um das okulomotorische System zu testen, eignet sich der Smooth Pursuit Neck Torsion Test (SPNT), der gleichmäßige Blickfolge Test in Rotation [21, 22]. Dabei zeigt Frau Meier bei rotiertem Rumpf und neutral gehaltener Kopfposition eine verlangsamte Augenbewegung und einen verstärkten Schwindel. Dies unterstützt die Hypothese des zervikogenen Schwindels und weist auf einen möglichen beitragenden Faktor des okulomotorischen Kontroll-Systems hin. Der SPNT scheint eine gewisse diskriminative Validität zur Unterscheidung zwischen zervikogener und vestibulärer Schwindelursache zu haben [23].

Auch die Joint Position Error Testung [21] mittels eines Laserpointers im Abstand von einem Meter zeigt im Sitzen ein Abwei-
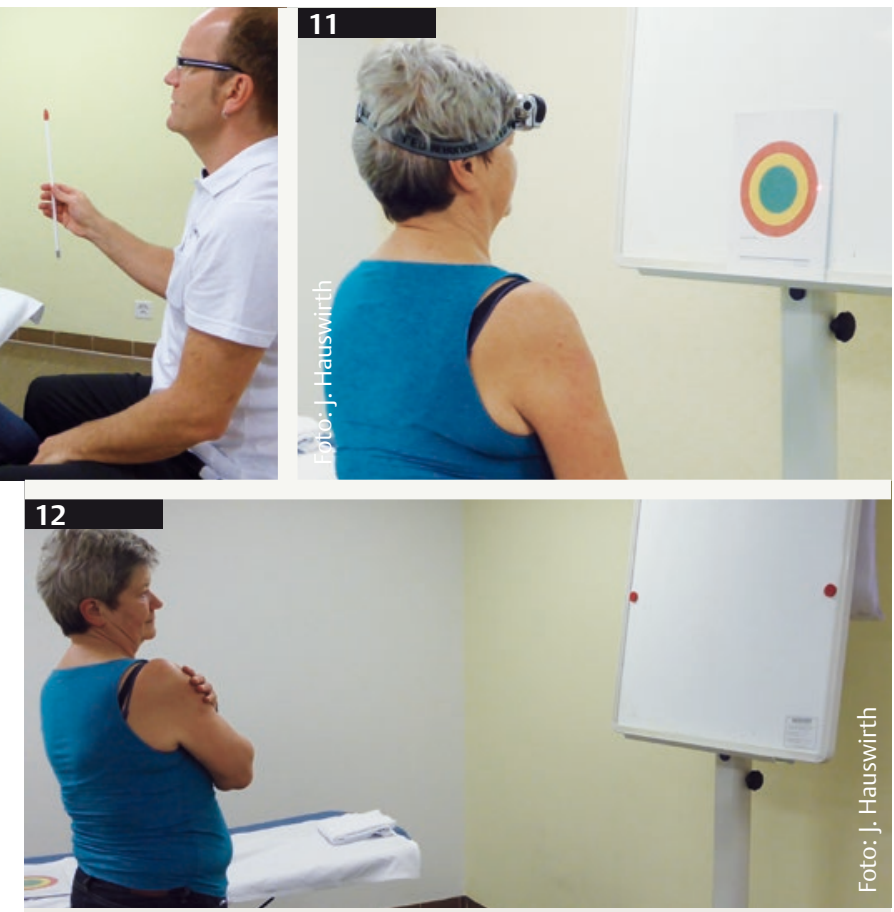

Abb. 9 SNAG Eigenmobilisation in Flexion C2.

Abb. 10 SPNT - Smooth Pursuit Neck Torsion Test.

Abb. 11 JPE - Joint Position Error Test.

Abb. 12 Okulomotorische Augenkontrollübungen in Rumpfrotation als Heimprogramm.

chen vom Startpunkt von über zehn Zentimeter in Rotations- sowie in Flexions- und Extensionsrichtung [23] ( $\bullet$ Abb. 11). Dieser Test sollte normalerweise keine Abweichungen über fünf Zentimeter zeigen.

Die Standsicherheit untersuche ich mit dem Romberg Test (Füsse zusammen) und im Tandem-Stand nach Treleaven et al. (2008) [23]. Beide Tests zeigen deutlich ein verstärktes laterales und sagittales Schwanken mit geschlossenen Augen.

Hypothesenbildung. Da der SPNT eine Veränderung der Augenbewegung zeigt, erhält Frau Meier zusätzlich eine Heimübung für die Augenbewegung. JPE und Rombergtest dienen als Verlaufszeichen.

\section{Sechste bis neunte Behandlung}

Diese vorläufig letzten Behandlungen beinhalten vor allem okulomotorische Kontrollübungen mittels Augenbewegungen im Stand. Frau Meier hat die Aufgabe, sprunghafte Augenbewegungen in verschiedenen Rotationsstellungen des Rumpfes auszuführen. Zur Hilfe klebt sie sich dazu zwei rote Punkte zu Hause an die Wand.

Da der JPE grösser als fünf Zentimeter Abweichung in Rotation und Flexion/Extension zeigte, ist die zweite Heimübung in dieser Phase eine Repositionsübung mit dem Laser zu Hause.

\section{Befund nach neun Behandlungen}

Die Beweglichkeit der HWS ist deutlich verbessert und ohne Schwindelreproduktion ( Tab. 4).

Die Schwindelhäufigkeit hat sich von täglich auf zweimal pro Woche, die Schwindelintensität auf VAS 1/10 verringert. Die Verbesserung des Schwindels gibt Frau Meier mit 90 Prozent an.

Der DHI ist weitgehend normalisiert auf 8/100. 
Tab. 4 Aktive Bewegungen HWS - alle ohne Schwindelauslösung.

\begin{tabular}{|l|l|}
\hline Bewegungsrichtung & Range of Motion \\
\hline Flexion & $50^{\circ} \square$ \\
\hline Extension & $50^{\circ} \square$, Knick in der mittleren HWS \\
\hline Rotation links & $70^{\circ} \square$ \\
\hline Rotation rechts & $70^{\circ} \square$ \\
\hline Lateralflexion links & $30^{\circ} \square$ \\
\hline Lateralflexion rechts & $30^{\circ} \square$ \\
\hline
\end{tabular}

Frau Meier zeigt eine sehr gute Adhärenz, übt weiterhin selbstständig ihr Heimprogramm und kann dadurch drei Wochen nach Behandlungsabschluss ihren Schwindel vollständig eliminieren.

\section{FAZIT}

○

Bei Frau Meier bestätigte sich die Hypothese des zervikogenen Schwindels, der durch ein multimodales Behandlungsmanagement beseitigt werden konnte. Eine hochzervikale manuelle Mobilisation verbesserte den muskuloskeletalen Anteil des Schwindels. Sensomotorisches Training und ein gezielter Muskelaufbau der tiefen Nackenflexoren eliminierten die funktionelle Instabilität der mittleren HWS, die ein beitragender Faktor war. Ein okulomotorisches Training und das konsequente Üben zu Hause beseitigten letztendlich den Schwindel ganz. Ein Jahr nach der Therapie berichtete Frau Meier am Telefon, dass dieses positive Ergebnis immer noch besteht.
Literaturverzeichnis am Ende der HTML-Version unter www.thieme-connect.de/products/manuelletherapie

\section{AUTOR}

Jürg Hauswirth aus Reinach bei Basel ist Manualtherapeut (PT MAS msk, OMT svomp®) und IMTA Senior-Instruktor. Er arbeitet klinisch am Kantonsspital Baselland Bruderholz und unterrichtet IMTA Kurse in Deutschland und der Schweiz. In

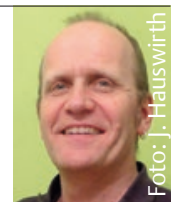
seiner Abschlussarbeit als Instruktor hat er sich eingehend mit dem Thema zervikogener Schwindel befasst. Bei dem Buch „Gleichgewicht und Schwindel“ von Stefan Schädler arbeitete er zudem als Co-Autor am Kapitel „Zervikogener Schwindel" mit [19]. Er behandelt mit Vorliebe Patienten mit muskuloskeletalen Beschwerden, insbesondere Nackenbeschwerden und Schwindel.

Physiotherapie Kantonsspital Baselland

4101 Bruderholz

Schweiz

juerg.hauswirth@imta.ch

\section{BIBLIOGRAFIE}

DOI 10.1055/s-0042-108663

manuelletherapie 2016; 20: 122-127

(c) Georg Thieme Verlag KG

Stuttgart · New York · ISSN 1433-2671

\section{Neue OMT-Weiterbildung der DGOMT e.V.}

Die Deutsche Gesellschaft für Orthopädische Manuelle Therapie (DGOMT e. V.) bietet ihre nächste OMT-Weiterbildung in Zusammenarbeit mit der Universität Freiburg und der Hochschule Furtwangen an. Die Weiterbildung startet am 27. August 2016 in München und setzt sich aus 2 Theoriemodulen (durchgeführt von der Universität Freiburg und der Hochschule Furtwangen), 5 Praxismodulen und der Supervision (Mentored Clinical Practice, MCP) zusammen. Dabei können insgesamt 70 ECTS-Punkte gesammelt werden, die Voraussetzung für die Zulassung zum OMTAbschlussexamen sind.

Physiotherapeuten, die bei der DGOMT e.V. die Internationale Weiterbildung erfolgreich absolviert haben, können diesen auf den Master-Studiengang „Interdisziplinäre Gesundheitsförderung“ anrechnen lassen. Diesen Studiengang bieten die Universität Freiburg und die Hochschule Furtwangen ab dem Wintersemester 2017/2018 an.
Der Master-Abschluss ist allerdings nur für Personen mit abgeschlossenem Bachelor-Studium möglich. Dazu sind zunächst ein Modul wissenschaftliches Arbeiten und ein Praktikum abzuschließen, bevor die Master-Thesis mit den dazugehörigen Prüfungen absolviert werden kann. Nach 3-5 Jahren (frei wählbar, da berufsbegleitend) erreichen Sie dann den Abschluss Master of Science in „Interdisziplinärer Gesundheitsförderung“ mit dem Schwerpunkt Biomechanik, Gesundheitsdiagnostik und Orthopädischer Manueller Therapie.

Nähere Informationen unter:

www.igf-studium.de od. www.dgomt.de.

Anmeldung für das Modul „Angewandte Orthopädische Manuelle Therapie" unter:

Sekretariat des OMT-Weiterbildungsträgers (DGOMT e.V.)

Nora Szüszner, Grünfeldstr. 8, 83026 Rosenheim

info@noraszueszner.de 